\title{
Dynamical System Analysis for Inflation with Dissipation
}

\author{
H. P. de Oliveira* and Rudnei O. Ramos ${ }^{\dagger}$ \\ Universidade do Estado do Rio de Janeiro, \\ Instituto de Física - Departamento de Física Teórica, \\ 20550-013 Rio de Janeiro, RJ, Brazil
}

August 1997

\begin{abstract}
We examine the solutions of the equations of motion for an expanding Universe, taking into account the radiation of the inflaton field energy. We then analyze the question of the generality of inflationary solutions in this more general setting of a dissipative system. We find a surprisingly rich behavior for the solutions of the dynamical system of equations in the presence of dissipational effects. We also determine that a value of dissipation as small as $\sim 10^{-7} H$ can lead to a smooth exit from inflation to radiation.
\end{abstract}

PACS number(s): $98.80 \mathrm{Cq}$

*E-mail: oliveira@symbcomp.uerj.br

${ }^{\dagger} \mathrm{E}$-mail: rudnei@symbcomp.uerj.br 


\section{Introduction}

It has been by now around 16 years since the idea of inflation [1] was introduced in order to solve some of the underlying problems of the standard big bang theory. Since then, inflation has become one of the most important paradigms of early Universe cosmology. The main idea of inflation is the existence of a period of accelerated expansion of the Universe, when its energy density becomes dominated by a potential energy density $V(\phi)$ of a scalar field $\phi$ (the inflaton). The dynamics of inflation depend on the specifics of the many models available describing inflation, but the basic mechanisms are based on the equation of motion of a (homogeneous) inflaton field, represented by its classical equation of motion

$$
\ddot{\phi}+3 H \dot{\phi}+V^{\prime}(\phi)=0,
$$

(overdot represent derivative with respect to time and $V^{\prime}=\frac{d V}{d \phi}$ ) together with those of gravity.

The basic assumption of most inflationary models is the one related with the so called slow-roll approximation: at some initial time $t_{0}$, the scalar field has a value far from the minimum of the potential $V(\phi)$. At this time the scalar field energy density dominates and the Universe enters the inflationary era. Sufficient inflation, required to solve the main problems of the standard big bang theory, demands that $\phi$ rolls down to the minimum sufficiently slowly. It is a common belief that during this stage the particle like matter components are all rapidly red-shifted away (as well the temperature, if we initially started in a thermal bath) and that the evolution of $\phi$ can be well represented by (1.1). Later, when $\phi$ hits the bottom of the potential and starts oscillating in a time scale shorter than the Hubble time, all the energy stored in the oscillating field $\phi$ is then released in the form of light particles that $\phi$ may be coupled to. These decay products then thermalize, reheating the Universe. This final stage of inflation is the so called reheating stage [for an account of new developments in the theory of reheating, see, for instance, [2] and references therein].

More recently, however, Berera and Fang [3] have shown that these two distinct stages of inflation can be compounded if the inflaton dissipates its energy into a thermal bath. They have shown that this new scenario is still consistent, providing sufficient e-folding of inflation and generation of density perturbations of the right magnitude, and that these density perturbations are consequences of thermal fluctuations, as opposite to quantum fluctuations, generated during the slow-roll stage, in the supercooled inflationary scenario. This new picture for inflation has been denominated warm inflation 国, 司.

In this modified description of inflation, we must alter (1.1) to take into account the continuous dissipation of the inflaton field in a thermal bath. A phenomenological way of doing this is, for example, by introducing a friction-like term $\eta(\phi) \dot{\phi}$ in (1.1). $\eta(\phi)$ is the dissipation coefficient that comes from the damping or "decay" of the scalar field $\phi$ interacting with an environment or bath degrees of freedom, constituted by, for example, 
light fields or field modes that $\phi$ may be coupled to. The form of $\eta(\phi)$ depends on the details of the interaction terms. The inclusion of a friction term in the equation of motion of the inflaton field, to study its evolution in the early stages of inflation, is not new. It has long been recognized that dissipative processes, associated with the inflaton decay during its evolution, are able to slow down the rolling of the field $\phi$ and, therefore, that these processes would be able to support inflation in inflationary models such as new or chaotic inflation [6]. Recent works on microscopic approaches to nonequilibrium dynamics of quantum fields [5, 7, 8] support the introduction of a friction term of the above form in the field equation of motion, especially under certain conditions, such as near-equilibrium and when a loop expansion is valid. These studies (see also, [9, 10]) have also demonstrated the generality of dissipation (as well as fluctuations) in the dynamics of fields in interaction with a bath (thermal or quantum). Thus, from the moment that we consider that the inflaton is coupled to light fields, which is a necessary condition for describing reheating, we must also consider the possible effects of backreactions of these fields, which include dissipation through the decay of the inflaton also during the stage of inflation.

In principle we may consider, among other things, that quantities such as sufficient inflation, the magnitude of initial density perturbations, the strength of coupling constants of the inflaton field with other fields during the inflationary era, not only constrain, but also dictate how important dissipation is during inflation. For instance it is known [11] that small coupling constants are necessary in order that the slow-roll method to be a valid approximation, as well as for obtaining the right magnitude of initial density perturbations. Equivalently, density perturbations constrain quantum fluctuations to remain small during the slow-roll stage of inflation. On the other hand, in the warm inflation

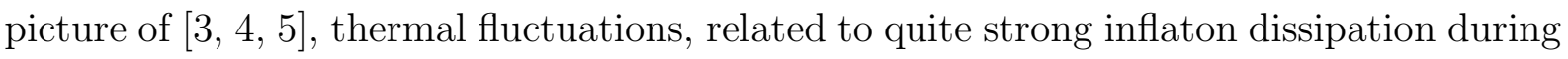
slow-roll, have been shown to be compatible with generation of initial density perturbations, with minimal requirements on the inflationary potential, besides that of the slow-roll approximation.

Recent developments in nonequilibrium dynamical processes in the early Universe, in particular during reheating, and the warm inflation picture, lead us to consider some basic questions concerning dissipation during inflation. Not only a proper microphysical approach is necessary to understand the appearance of a non-negligible inflaton dissipation at the early stages and/or during inflation, but we also need to investigate how the classical inflaton dynamic gets changed in a dissipative environment. In this paper we will be concerned with the latter question, since it can shed some light about how specific prescriptions for dissipation may change the dynamics in a pre-inflationary era, and then, possibly, help to constrain field theoretic model candidates to describe inflation in this new setting. A detailed microscopic model will be presented in a forthcoming paper. The qualitative analysis of the resulting set of field equations is the first step in understanding radiation energy properties of the Universe and in discriminating how general inflation is in dissipative systems. Such analysis includes the asymptotic behavior of the solutions 
near the critical points representing the initial singularity and the basic features of the trajectories in the phase space that will be important for the choice of initial conditions used in the numerical experiments. We perform this study in the framework of the theory of dynamical systems [12]. We then generalize earlier works on this subject [see the Refs. [13, 14]], which were previously done in terms of the classical set of equations for gravity plus that of the inflaton field $\phi$, without dissipation. This study is important both to understand the dynamics of inflation in a scenario like warm inflation, as it is also an example of a dissipative dynamical system, which may have other important applications in early Universe cosmology.

The paper is organized as follows: In Sec. II we give the basic equations, define the region in phase space of interest to our study and discuss some of their properties for different kinds of dissipation. In Sec. III we present an analysis of the dynamical system of equations in the presence of dissipation and determine the relevant asymptotic solutions of the system of equations. In Sec. IV we study how dissipation changes the number of physically relevant (advantageous) trajectories, i.e., those having, e.g., a long enough inflationary stage. We also give limiting values of when dissipation is of relevance in the inflationary scenario. Sec. $\mathrm{V}$ is devoted to discussions of our results and conclusions.

\section{The Dynamical System}

We start by writing the basic equations that characterize our dynamical system:

$$
\begin{gathered}
H^{2}=\frac{8 \pi G}{3}\left(\rho_{\phi}+\rho_{\mathrm{rad}}\right)-\frac{k}{a^{2}}, \\
2 \dot{H}+3 H^{2}+\frac{k}{a^{2}}=-8 \pi G\left(p_{\phi}+p_{\mathrm{rad}}\right), \\
\ddot{\phi}+3 H \dot{\phi}+V^{\prime}(\phi)+\eta(\phi) \dot{\phi}=0,
\end{gathered}
$$

where $H=\dot{a} / a$ is the Hubble parameter and $G=1 / M_{\mathrm{pl}}^{2}$, with $M_{\mathrm{pl}}$ the Planck mass. $k=0,+1,-1$ for a flat, closed or open Universe, respectively. $\rho_{\phi(\mathrm{rad})}$ and $p_{\phi(\mathrm{rad})}$ are the energy density and pressure for $\phi$ (radiation), respectively. We also have the standard

relations: $\rho_{\phi}=\frac{1}{2} \dot{\phi}^{2}+V(\phi), p_{\phi}=\frac{1}{2} \dot{\phi}^{2}-V(\phi)$ and $p_{\text {rad }}=\frac{1}{3} \rho_{\text {rad }}$. Equations (2.1) and (2.2) are the standard gravity equations derived from the Einstein equations. Eq. (2.3) is the equation of motion for the (homogeneous) scalar inflaton field $\phi$, with $V(\phi)$ the effective potential for $\phi$. We have introduced dissipation through a friction term $\eta(\phi) \dot{\phi}$ in the equation of motion.

Equation (2.3) deserves some important considerations. First, in a realistic model the field dependence of the friction term may not be exactly like the one given in (2.3), particularly in highly non-equilibrium situations and in a time dependent background, as some recent studies have indicated [10]. It has been demonstrated [0, 8], however, 
that $\eta(\phi) \dot{\phi}$ describes quite well the dissipation of the field $\phi$ in a bath environment in near-equilibrium and when a perturbative expansion is valid. In spite of this and without a better understanding from a field theoretic point of view of nonequilibrium systems , we take for simplicity the friction term in the form given in (2.3). Since our analysis is mostly qualitative, we expect that we will not miss much of the important behavior of the system given a different prescription for dissipation. Secondly, we take $\eta(\phi)$ as a polynomial in $\phi$. This is the case for standard polynomial interactions among fields, at least when a loop expansion in the fields is valid. The field dependence of $\eta(\phi)$ is highly dependent of the type of interaction we consider of the scalar field $\phi$ with the fields (or modes) that describe the bath environment. For example, $\eta(\phi)$ may in some approximations be well represented by a constant in the case of linear couplings (like $\phi \psi)$ [5], or be quadratic in $\phi$ for quadratic couplings (like $\phi^{2} \psi^{2}$ ) [7]. $\eta(\phi)$ may also have a nontrivial dependence on coupling constants and temperature, as is the case at high temperatures, which is the situation of interest in warm inflation scenarios. Further considerations on $\eta(\phi)$ will be considered below. Finally, Eq. (2.3) must be viewed as the equation obtained by averaging over a noise field, which is directly related to dissipation through some generalized fluctuation-dissipation relation. This means we are considering the inflaton field as a classical background [8] and we are looking at the averaged behavior of the inflaton field in time. This is a good approximation as far as we look to the inflaton dynamics at a sufficiently long period of time when compared to the typical "wavelength" of the fluctuations, given by $\sim\left[V^{\prime \prime}\right]^{-1 / 2}$. Our scale of time will be basically the duration of inflation, which is at least 20 times larger than that value (for e-folds of inflation larger than 70), as we will see in Sec. IV. Also, the amplitude of fluctuations, given by the noise field two-point correlation function, should not be too large during the inflaton evolution, implying, roughly, that our approximation of taking the ensemble averaged equation of motion is better the smaller is the dissipation. Keeping in mind these considerations, we proceed with the definition of the equations representing our dynamical system.

We have that $\rho_{\phi}$ and $\rho_{\text {rad }}$ evolve in time as [1]:

$$
\begin{gathered}
\dot{\rho}_{\phi}+3 H \dot{\phi}^{2}+\eta(\phi) \dot{\phi}^{2}=0 \\
\dot{\rho}_{\mathrm{rad}}+4 H \rho_{\mathrm{rad}}-\eta(\phi) \dot{\phi}^{2}=0 .
\end{gathered}
$$

Assuming a flat Universe $(k=0)$, from (2.1), we can consider

$$
\rho_{\mathrm{rad}}=\frac{3}{8 \pi G} H^{2}-\rho_{\phi}=\frac{3}{8 \pi G} H^{2}-\frac{1}{2} \dot{\phi}^{2}-V(\phi)
$$

as the first integral of (2.5). Using $p_{\phi}$ and $p_{\text {rad }}$ in (2.2), we can write for $\dot{H}$ the equation:

$$
\dot{H}=-2 H^{2}-\frac{8 \pi G}{6}\left(\dot{\phi}^{2}-4 V(\phi)\right) .
$$


The equations (2.3), (2.7), together with $\dot{\phi}=\frac{d \phi}{d t}$, define a three-dimensional (dissipative) dynamical system in the phase space of $(\phi, \dot{\phi}, H)$.

For definitiveness, we take the simplest potential $V(\phi)$, which describes a massive scalar field $\phi$ : $V(\phi)=\frac{1}{2} m^{2} \phi^{2}$. The generalization of our results for more complicated potentials, including self-interactions terms and symmetry breaking, should not present too much difficulty. Next, we take $\eta(\phi)$, as discussed later, in the form $\eta(\phi)=\eta_{n} \phi^{n}$. We can further constrain the form of $\eta(\phi)$ by noting that the friction coefficient must be positive definite due to entropy requirements and the parity invariance of the potential. Thus, $\eta_{n}>0$ and $n=0$ or an even integer. We analyze two main cases of possible interest: that of $n=0$, in which case $\eta$ reduces to a constant; and that of $n=2$, when $\eta \propto \phi^{2}$. Substituting $V$ and $\eta$ in (2.3) and (2.7) and redefining variables $t \rightarrow t / m, \phi \rightarrow \frac{1}{\sqrt{8 \pi G}} x$, $\dot{\phi} \rightarrow \frac{m}{\sqrt{8 \pi G}} y, H \rightarrow m z, \eta_{0} \rightarrow m \beta_{0}$ and $\eta_{2} \rightarrow 8 \pi G m \beta_{2}$, we obtain the dynamical system:

$$
\begin{aligned}
\dot{x} & =y \\
\dot{y} & =-3 y z-x-\beta_{n} x^{n} y \\
\dot{z} & =-2 z^{2}-\frac{1}{6} y^{2}+\frac{1}{3} x^{2} .
\end{aligned}
$$

The physical region in the phase space $(x, y, z)$ is defined by the condition $\rho_{\text {rad }} \geq 0$ which, in the units of (2.8), corresponds to:

$$
3 z^{2}-\frac{1}{2} y^{2}-\frac{1}{2} x^{2} \geq 0,
$$

or, expressing the above relation for $z, z^{2} \geq \frac{1}{6}\left(x^{2}+y^{2}\right)$, which defines a conical volume in $(x, y, z)$, with the physical region $\rho_{\text {rad }} \geq 0$ inside the cone. Expanding universes $(H>0)$ correspond to those trajectories for which $z>0$ (inside the upper half of the conical volume). For our simple quadratic potential there is only one critical point, namely, the origin that represents the Minkowski spacetime. In general it is an attractor and the form of approach depends upon the dissipation term. In fact we can distinguish two main types of approach depending on the kind of dissipation we may have. If $n=0$, we can readily show from (2.8) that the trajectories on the physical region approach the origin in the direction given by the $z$-axis. The $z$-axis itself represents the usual Friedmann model with pure radiation and is, in this case, an attractor for the late stages of field evolution. If $\beta_{0}<2$ the approach to the $z$-axis is oscillatory, while for $\beta_{0} \geq 2$ it is exponential. If $n=2$ the approach is always oscillatory tending to the origin on the surface $\rho_{\text {rad }}=0$. In Figs. 1 (a) to 1(c) we plot the field trajectories by a direct numerical integration of the system (2.8) for some representative values of initial conditions and dissipative coefficients.

In the absence of dissipation, the conical surface $\rho_{\text {rad }}=0$ is an invariant manifold. As a consequence, all initial conditions chosen on this surface will produce trajectories that evolve on it and end at the origin. In particular, the dynamical system for which $\rho_{\text {rad }}=0$ 
has been analyzed by Belinskii et. al. [13, 14. The effect of dissipation is to destroy the invariant manifold since

$$
\left.\dot{\rho}_{\text {rad }}\right|_{\rho_{\text {rad }}=0}=\eta(\phi) \dot{\phi}^{2} \neq 0 \text {. }
$$

In this case the trajectories may no longer lie completely in the surface $\rho_{\text {rad }}=0$, even if the initial conditions are taken on it. An important question we can ask is: in which way do the curves cross the surface $\rho_{\text {rad }}=0$ ? A simple way to answer this question is, for example, by taking the scalar product at $\rho_{\text {rad }}=0$ between the vector normal to the outside surface of the cone, $\vec{n} \propto-\vec{\nabla} \rho_{\text {rad }}=(x, y,-6 z)$, with the velocity field vector of the trajectories on phase space, $\vec{v}=(\dot{x}, \dot{y}, \dot{z})$ :

$$
\vec{n} .\left.\vec{v}\right|_{\rho_{\mathrm{rad}}=0} \propto-\beta_{n} x^{n} y^{2} .
$$

Since we are considering $\beta_{n}>0$ and $n$ even, we obtain that $\left.\vec{n} \cdot \vec{v}\right|_{\rho_{\text {rad }}=0}<0$, which means that those trajectories that initially lie inside the cone $\left(\rho_{\mathrm{rad}}>0\right.$ region $)$ remain inside the cone. The only trajectories that can cross the cone lie initially outside it. This is a consequence of the requirement of $\eta(\phi)$ be always positive definite. In particular we note that for an odd integer $n$, we may have (2.11) positive for $x<0$ and we could have trajectories leaving the region $\rho_{\text {rad }}>0$ to $\rho_{\text {rad }}<0$, in violation of the second law of thermodynamics.

The inflationary region in phase space is defined by

$$
\frac{\ddot{a}}{a}=\dot{H}+H^{2}>0
$$

which, in terms of $x, y$ and $z$, gives

$$
-z^{2}-\frac{1}{6} y^{2}+\frac{1}{3} x^{2}>0 .
$$

Trajectories satisfying (2.13) are the ones that are in the inflationary regime. The intersection between the regions defined by (2.9) and (2.13) defines the inflationary physical region of interest to us.

\section{Analysis at Infinity and Asymptotic Solutions}

The analysis at infinity provide us with important information concerning the behavior of solutions in the region where $x^{2}+y^{2}+z^{2} \rightarrow \infty$. Such a region contains information about the early stages of the universe just after the quantum domain, and therefore can be described by the classical equations. An important issue to be discussed is whether inflation plays the role of an attractor for most of solutions no matter the presence of dissipation. For our work, the following coordinate transformation is found to be useful: 


$$
x=\frac{u}{w}, y=\frac{v}{w}, z=\frac{1}{w},
$$

where infinity is represented by the invariant manifold $w=0$. The projections of the physical region $\rho_{\text {rad }} \geq 0$ as well as the inflationary domain $\frac{\ddot{a}}{a}>0$ on this plane, yield, respectively, the regions described as $u^{2}+v^{2} \leq 6$ and $2 u^{2}-v^{2} \geq 0$. The idea underlying the choice of coordinates (3.1) is to compactify infinity, so that a dynamical system in these variables describes properly the basic features of the asymptotic behavior of the solutions. In Fig. 2, we present a better view of the compactified region given by the cap of the conical region.

The dynamical system (2.8) in the coordinates $u, v, w$ is

$$
\begin{aligned}
& \frac{d u}{d \tau}=u w^{\frac{n}{2}}\left(2+\frac{v^{2}}{6}-\frac{u^{2}}{3}\right)+v w^{\frac{n}{2}+1} \\
& \frac{d v}{d \tau}=v w^{\frac{n}{2}}\left(-1+\frac{v^{2}}{6}-\frac{u^{2}}{3}\right)-u w^{\frac{n}{2}+1}-\beta_{n} u^{n} v w^{1-\frac{n}{2}} \\
& \frac{d w}{d \tau}=w^{\frac{n}{2}+1}\left(2+\frac{v^{2}}{6}-\frac{u^{2}}{3}\right)
\end{aligned}
$$

where we have changed the time parameter in such a way that $d t=w^{\frac{n}{2}+1} d \tau$, which is valid for $n=0,2$. The critical points at infinity lie on the plane $w=0$ and their nature depend on the type of dissipation we are considering. They represent the initial singularity since, from Eqs. (3.5)-(3.8) below, we see that the scale factor goes to zero at these points. In addition, at the critical points the scalar of curvature $R=6\left(\dot{H}+2 H^{2}\right)=\frac{m^{2}}{w^{2}}\left(2 u^{2}-v^{2}\right)$ diverges. As stated before two cases will be analyzed, namely, $n=0$, for constant dissipation, and $n=2$, for field dependent dissipation.

\section{III.a Case $\mathbf{n}=0$}

We adopt the following steps here and for the case $n=2$ to analyze the region at infinity $x^{2}+y^{2}+z^{2} \rightarrow \infty$. First, we determine all critical points on the surface $w=0$ that are located in the region defined by $0 \leq \theta \leq \theta^{*}$ (cf. Fig. 2) $\left(\tan \theta^{*}=\sqrt{6}\right)$, or in terms of the new coordinates (3.1), inside the region $u^{2}+v^{2} \leq 6$. Secondly, in performing the stability analysis, we may obtain approximate solutions near those critical points and lying on the physical region. Thirdly, the knowledge of unphysical trajectories that lie entirely on the surface $w=0$ is important since, by continuity arguments, such trajectories determine the behavior of the integral curves of the system that lie within the cone but near its surface 14 .

After substituting $n=0$ into the system (3.2) and taking $w=0$, a two-dimensional system arises 


$$
\begin{aligned}
& \frac{d u}{d \tau}=u\left(2+\frac{v^{2}}{6}-\frac{u^{2}}{3}\right) \\
& \frac{d v}{d \tau}=v\left(-1+\frac{v^{2}}{6}-\frac{u^{2}}{3}\right) .
\end{aligned}
$$

Five critical points are found: two repelling nodes $P_{2}(u=0, v=\sqrt{6}), P_{2}^{\prime}(u=0, v=$ $-\sqrt{6})$, one saddle $P_{0}(u=0, v=0)$, and $P_{1}(u=\sqrt{6}, v=0), P_{1}^{\prime}(u=-\sqrt{6}, v=0)$ which are degenerate. In Fig. 3, these points are shown on the plane $w=0$ together with the unphysical trajectories on it. Note that the projection of the cone $\rho_{\text {rad }}=0$ onto the plane $w=0$ is an integral curve of the system (3.3). The regions near $P_{1}, P_{1}^{\prime}$ are the projections of the inflationary domain $2 u^{2}-v^{2} \geq 0$ on $w=0$. According to the phase portrait shown in Fig. 3, the inflationary domain is an attractor for most trajectories. Actually this is a general feature of the system, as can be seen from Fig. 4, where we have plotted $\frac{\ddot{a}}{a}$ as a function of time. Initial values were purposefully taken away from the inflationary region. Note how the system is driven to the inflationary domain in the different cases of dissipation.

Curves emanate from $P_{2}$ and $P_{2}^{\prime}$ to the interior of the cone. The asymptotic behavior near these points is

$$
H=\frac{1}{3 t}, \quad \phi= \pm \sqrt{\frac{3}{4 \pi}} M_{\mathrm{pl}} \ln (m t)+\frac{v_{0} M_{\mathrm{pl}}}{2 \sqrt{8 \pi}}(m t)^{\frac{2}{3}} \mp \frac{\beta_{0}}{m M_{\mathrm{pl}}} t,
$$

where $v_{0}$ is an arbitrary constant and the signs \pm indicate which critical point $P_{2}, P_{2}^{\prime}$ we are considering. The emergence from these points corresponds to the growth of time $t$ from the instant $t=0$ of the initial singularity. Near these singularities, $\dot{\phi}^{2} \gg \phi^{2}$, meaning that the kinetic energy of the scalar field is dominant over the potential $V(\phi)$ producing an effective equation of state $p_{\phi}=\rho_{\phi}$. Therefore all curves emanating from $P_{2}, P_{2}^{\prime}$ are not in the inflationary regime. Note that the influence of dissipation starts to be considerable as far as the trajectories evolve away from the critical points. It is also possible to integrate the dynamical system near the critical point $P_{0}$, whose asymptotic behavior near it is characterized by

$$
H=\frac{1}{2 t}, \quad \phi= \pm \frac{M_{\mathrm{pl}}}{2 \sqrt{\pi m t}},
$$

where the initial singularity is denoted by $t=0$.

The points $P_{1}, P_{1}^{\prime}$ lie on the inflationary region and are attractors of trajectories on the invariant manifold $w=0$, suggesting that they are also attractors for trajectories on the physical region but close to the invariant manifold. It can be shown that there is only one trajectory emerging from $P_{1}$ and $P_{1}^{\prime}$ into the physical region, whose asymptotic solution is 


$$
H=-\frac{1}{3} m t, \quad \phi= \pm \frac{m M_{\mathrm{pl}}}{\sqrt{8 \pi}} t
$$

where $t$ increases from $-\infty$, the initial singularity. As expected, this solution is inflationary, since $m^{2} \phi^{2} \gg \dot{\phi}^{2}$, which produces an effective equation of state $p_{\phi}=-\rho_{\phi}$. All remaining trajectories that visit a small neighborhood of these points experience an inflationary phase as described above. It is worth pointing out that the asymptotic solution

(3.6) corresponds to the direction of approach given by $v=-\frac{\sqrt{6}}{3} w$, which is equivalent to no dissipation (see analysis carried out by Belinskii et al[14]). The influence of dissipation, from a purely dynamical system analysis, appears as a second order effect changing the former relation to $v=-\frac{\sqrt{6}}{3} w+\frac{\sqrt{6}}{9} \beta_{0} w^{2}$.

\section{III.b Case $n=2$}

The structure of the phase space for $n=2$ is quite different from the previous case. After substituting $n=2$ into the system (3.2), we obtain a two-dimensional dynamical system whose phase portrait is shown in Fig. 5. There are two lines of degenerate critical points, denoted by $l_{1}(-\infty<u<\infty, v=w=0)$ and $l_{2}(-\infty<v<\infty, u=w=0)$. Here the projection of the cone surface $\rho_{\text {rad }}=0$, represented by the dashed circle on Fig. 5, is not an integral curve of the system at infinity. By continuity arguments, taking into account the unphysical trajectories on $w=0$, we infer that the trajectories within the physical region, but near the plane $w=0$, originate outside the region $\rho_{\text {rad }}=0$, eventually outside the quantum domain, i.e., where the classical description is valid. Therefore, they must be ruled out of our analysis. Nonetheless, some critical points on the lines $l_{1}, l_{2}$ may be source of trajectories in the physical region. In fact, using standard methods to analyze sets of degenerated critical points [12], we come to the conclusion that the points at infinity $P_{0}(u=v=0), P_{1}(u=\sqrt{6}, v=0), P_{1}^{\prime}(u=-\sqrt{6}, v=0), P_{2}(u=0, v=\sqrt{6})$, $P_{2}^{\prime}(u=0, v=-\sqrt{6})$ are the relevant ones and belong to a distinct nature from the remaining point, as we are going to describe. From the points $P_{0}, P_{2}$ and $P_{2}^{\prime}$ threedimensional pencils of trajectories emerge, whose asymptotic behavior near the points $P_{0}$ and $P_{2}, P_{2}^{\prime}$, are given, respectively, by

$$
\begin{gathered}
H=\frac{1}{2 t}, \quad \phi= \pm \frac{M_{\mathrm{pl}}}{2 \sqrt{\pi m t}}, \\
H=\frac{1}{3 t}, \quad \phi= \pm \sqrt{\frac{3}{4 \pi}} M_{\mathrm{pl}} \ln (m t) .
\end{gathered}
$$

In the above solutions the initial singularity is characterized by $t=0$, or equivalently the points $P_{0}$ and $P_{2}, P_{2}^{\prime}$, and the emergence from the singularity corresponds to increase of time from this value. We remark that the solutions are not inflationary, but eventually evolve to this stage. 
The most interesting class of solutions emanate from the points $P_{1}, P_{1}^{\prime}$ on the line $l_{1}$ (see Fig. 5). It can be shown that a two-dimensional pencil of paths emerges from these points along the same plane of the line $l_{1}$ and orthogonal to the plane $w=0$. The asymptotic behavior in this region is characterized by $v=0$, which implies that the form of the solutions is the same as found in (3.6).

\section{Advantageous Trajectories and Radiation in Inflation with Dissipation}

We now have a closer look at those trajectories in phase space that may lead to a long enough inflationary stage and let us try to quantify these trajectories in a way similar to that in [13]. During an inflationary period given by $\Delta t=t_{f}-t_{i}$, the scale factor changes by

$$
\frac{a\left(t_{f}\right)}{a\left(t_{i}\right)}=\exp \left(\int_{t_{i}}^{t_{f}} H(t) d t\right) .
$$

Let us determine the above ratio in terms of the initial and final values for the scalar field, $\phi_{i}$ and $\phi_{f}$, respectively.

First note that the inflationary epoch can be characterized by those trajectories that satisfy

$$
m \phi \gg|\dot{\phi}| .
$$

We consider the slow-roll approximation which, in the presence of dissipation, can be written as

$$
\dot{\phi} \simeq-\frac{m^{2} \phi}{3 H+\eta(\phi)} .
$$

We also assume that $\rho_{\phi} \gg \rho_{\text {rad }}$ (see [3] and [5]). Then, from (2.1) and (4.2), we can write

$$
H \simeq \sqrt{\frac{4 \pi}{3}} \frac{m|\phi|}{M_{\mathrm{pl}}} .
$$

Using (4.4) and (4.3) in (4.1), we get the explicit expression:

$$
\frac{a\left(t_{f}\right)}{a\left(t_{i}\right)} \simeq \exp \left[\frac{2 \pi}{M_{\mathrm{pl}}^{2}}\left(\phi_{i}^{2}-\phi_{f}^{2}\right)-\sqrt{\frac{4 \pi}{3}} \int_{\phi_{i}}^{\phi_{f}} \frac{\eta(\phi)}{m M_{\mathrm{pl}}} d \phi\right] .
$$

For $\eta(\phi)=0$ we recover the usual expression for the scale factor ratio [1, 13]. Taking $\eta(\phi)=\eta_{n} \phi^{n}, n=0,2$, we finally obtain 


$$
\frac{a\left(t_{f}\right)}{a\left(t_{i}\right)} \simeq \exp \left[\frac{2 \pi}{M_{\mathrm{pl}}^{2}}\left(\phi_{i}^{2}-\phi_{f}^{2}\right)+\sqrt{\frac{4 \pi}{3}} \frac{\eta_{n}}{m M_{\mathrm{pl}}} \frac{\left(\left|\phi_{i}\right|^{n+1}-\left|\phi_{f}\right|^{n+1}\right)}{n+1}\right] .
$$

The term in the above exponential gives the total number, $N$, of e-folds during inflation. We have numerically checked the validity of the above equation. The physically interesting trajectories are as usual those with $N \gtrsim 70$ [1].

With no dissipation, $\eta_{n}=0, N \sim 70$ can be accomplished with $\phi_{i} \approx 3.4 M_{\mathrm{pl}}$ and $\phi_{f} \approx 0.2 M_{\mathrm{pl}}$ [1]. In the presence of dissipation, for a fixed $N$, dissipation decreases the values of both $\phi_{i}$ and $\phi_{f}$. For the values of $\phi_{i}$ and $\phi_{f}$, which $N \sim 70$ with $\eta_{n}=0$, we see from (4.6) that dissipation begins to change considerably $N$, say by more than $10 \%$, for $\eta_{0} \gtrsim m$, or $\eta_{2} \gtrsim 0.25 m / M_{\mathrm{pl}}^{2}$ (in terms of the dimensionless factors $\beta_{n}, \beta_{0} \gtrsim 1$ and $\beta_{2} \gtrsim 0.01$, respectively). In both cases we have an initial dissipation of order $\eta(\phi) \gtrsim m$. Since $H \approx m$, we must have $H \lesssim \eta(\phi)$ in order for dissipation to begin changing the inflationary phase considerably.

The authors in [13, 14] have characterized the measure of disadvantageous trajectories $(N<70)$ as $\sim m / M_{\mathrm{pl}}$, obtained by the ratio of the trajectories beginning at $|\phi|<\phi_{i}$, in relation to the complete quantum boundary, of length $2 \pi M_{\mathrm{pl}}^{2} / m$. Since for fixed $N$ the effect of dissipation is to reduce $\phi_{i}$, we find that in the presence of dissipation $m / M_{\mathrm{pl}}$ $\left(\sim 10^{-7}\right)$ is the upper bound for the measure of disadvantageous trajectories. Dissipation turns inflation even more general.

We can also evaluate the energy of the inflaton field transferred to radiation during the inflationary stage, from, for example, Eq. (2.5). In Fig. 6 we plot $\rho_{\text {rad }}$ against time for four different values of $\beta_{n}$. The mark indicates when the trajectories leave the inflationary region ( $\ddot{a} / a$ becomes negative). We find that there is a value of $\beta_{n}$ for which radiation is maximal, at the exit of the inflationary stage, given by $\beta_{0}, \beta_{2} \approx 1\left(\eta_{0} \approx m\right.$ and $\left.\eta_{2} \approx 25 \mathrm{~m} / M_{\mathrm{pl}}^{2}\right)$. These results are fairly independent of the initial conditions. Also, we can find an ideal range for $\beta_{n}$ such that we exit inflation smoothly to the radiation era with the Universe sufficiently hot. At thermalization

$$
\rho_{\mathrm{rad}}=\frac{\pi^{2} g_{*}}{30} T^{4}
$$

where $g_{*}\left(\sim 10^{2}-10^{3}\right)$ is the effective number of degrees of freedom. If we impose that at the end of inflation we should have at least $T \sim m\left(m \simeq 5.10^{-7} M_{\mathrm{pl}}\right.$ for the quadratic potential model [1]), we have numerically obtained, for both $\beta_{0}$ and $\beta_{2}$, the approximate bound:

$$
10^{-7} \lesssim \beta_{n} \lesssim 800
$$

Too high values of $\beta_{n}$ lead to a longer inflationary period. Most of the inflaton's energy is transferred to radiation which is red-shifted away before the end of inflation. There is no possibility of a reheating phase for these cases. On the other hand, very small values 
of $\beta_{n}$ can provide a smooth exit from inflation to radiation and the inflaton field energy

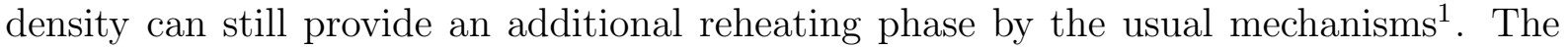
maximum temperature reached by the thermal bath, at the exit of the inflationary stage in our model, is $T \approx 10^{15} \mathrm{GeV}$, for $\beta_{0}, \beta_{2} \approx 1$.

\section{Discussions and Conclusions}

We have examined a dynamical system describing inflaton dynamics in the presence of dissipation. Dissipation is taken as being present throughout the inflaton's evolution and we have presented the changes in the phase diagram due to the effect of dissipation. Our results allow us to draw a series of important conclusions concerning the dynamical system and the physical system itself.

We have shown that the continuous production of radiation due to dissipation changes effectively the inflaton dynamical system. We have distinguished two main consequences for the field trajectories in phase space due to the presence of dissipation: (a) In field independent dissipation (for $\beta_{0} \geq 2$ ) the trajectories tend towards the $z$-axis at the final stages of evolution. In this regime the inflaton's motion is overdamped. The stronger the dissipation $\left(\beta_{0} \gg 2\right)$ the faster the field trajectories are attracted to the $z$-axis; (b) In field dependent dissipation the motion is initially damped but the field trajectories always tend to the cone surface $\rho_{\text {rad }}=0$. The stronger the dissipation the slower the field trajectories tend to this surface.

From the physical system side we can also reach some interesting conclusions which may have important consequences for inflationary dynamics. We have seen that dissipation associated with inflaton decay not only supports inflation but also draws all inflationary field trajectories into a longer stage of inflation. This is true for both kinds of dissipation we have considered. In terms of a measure of the amount of trajectories possessing the required inflationary period, a similar analysis to the one given in [13, 14] allows us to reach the conclusion that inflation is even more general in the presence of dissipation.

Our results also allow us to reach some conclusions concerning warm inflation scenarios or non-isentropic inflation, in particular concerning the problem of reheating. In nonisentropic inflation, as opposed to standard inflation, there is the possibility of no reheating phase, due to the continuous production of entropy and heat, leading to a smooth exit from inflation to the radiation era [5, 15]. We then expect that in these scenarios there is a strong damping of the inflaton field's motion in the direction of the minimum of the inflaton's potential. In this paper we have quantified how strong the dissipation must be, when compared to the friction due to expansion, in order to achieve favorable scenarios of radiation at the exit from the inflationary stage. We have shown that no reheating is only

\footnotetext{
${ }^{1}$ At least for $\beta_{0}<2$, when we are in the underdamped regime (see Sec. II) and for any value of $\beta_{2}$ smaller than the upper value in (4.8).
} 
achieved in the overdamped regime $\left(\beta_{0}>2\right)$ for constant dissipation, when there is no oscillatory motion of the inflaton around the potential minimum, or for very high values of $\beta_{2}$, when all the inflaton's energy is transfered to radiation and this is then red-shifted away due to the longer expansion.

A further question we may ask is about a possible realization of chaotic behavior in Cosmology. According with some previous in the realm of isotropic and anisotropic Bianchi IX cosmologies [16], the Universe can experience two possible outcomes: collapse into the big crunch after an initial stage of expansion and expansion into the inflationary regime. In this way, chaotic behavior is associated with an indetermination of the final state (collapse or expansion) of the Universe once a set of initial conditions in an infinitesimal region is chosen. We may say that the boundaries of collapse and expansion are mixed. In our casef all orbits initially in the physical region $\rho_{\text {rad }} \geq 0$ are unavoidably attracted to the state of unlimited expansion represented by the origin of the phase space (cf. Fig. 1). The orbits can not randomically explore the region inside the cone $\rho_{\text {rad }}=0$, since the volume of the phase space is not conserved. Therefore, we can conclude that chaotic behavior does not take place for those orbits lying in the physical region in the cases studied previously, i. e., for $n=0,2$. Such conclusions are also valid for the case analyzed by Belinskii and Khalatnikov [14 in the realm of anisotropic models.

Finally one of the most important results of this paper was to show that even a value of dissipation as small as $\eta(\phi) \sim 10^{-7} m$ (compare with $H \sim m$ ) can still lead to very important consequences for inflationary dynamics, such as providing a smooth exit from inflation to radiation, and it also shows that the interaction of the inflaton field with other degrees of freedom, manifested in the form of dissipation, cannot in general be neglected. We are currently working on a detailed microscopic model for dissipation in inflation, to be presented in a forthcoming paper.

\section{Acknowledgements}

We would like to thank D. Boyanovsky and A. Berera for useful discussions. We also thank J. E. Skea for reading and helping us to revise the text. This work was partially supported by Conselho Nacional de Desenvolvimento Científico e Tecnológico - CNPq (Brazil).

\section{References}

\footnotetext{
${ }^{2}$ Also similar conclusions are reached for the model studied by Belinskii et.al. [13, 14.
} 
[1] E. W. Kolb and M. S. Turner, The Early Universe (Addison-Wesley, Reading, MA 1990); A. D. Linde, Particle Physics and Inflationary Cosmology (Harwood, Academic, Chur, Switzerland 1990).

[2] L. Kofman, A. Linde and A. A. Starobinskii, Phys. Rev. D56, 3258 (1997).

[3] A. Berera and L. Z. Fang, Phys. Rev. Lett. 74, 1912 (1995).

[4] A. Berera, Phys. Rev. Lett. 75, 3218 (1995).

[5] A. Berera, Phys. Rev. D54, 2519 (1996).

[6] A. Albrecht, P. J. Steinhardt, M. S. Turner and F. Wilczek, Phys. Rev. Lett. 48, 1437 (1982); J. Yokoyama and K. Maeda, Phys. Lett. 207B, 31 (1988).

[7] M. Gleiser and R. O. Ramos, Phys. Rev. D50, 2441 (1994); M. Morikawa, Phys. Rev. D33, 3607 (1986); A. Hosoya and M. Sakagami, Phys. Rev. D29, 2228 (1984).

[8] D. Boyanovsky, H. J. de Vega, R. Holman, D. -S. Lee and A. Singh, Phys. Rev. D51, 4419 (1995).

[9] B. L. Hu, J. P. Paz and Y. Zhang, in The Origin of Structure in the Universe, edited by E. Gunzig and P. Nardone (Kluwer Academic, Norville, 1993); B. L. Hu, J. P. Paz and Y. Zhang, Phys. Rev. D45, 2843 (1993); ibid D47, 1576 (1993); E. Calzetta and B. L. Hu, Phys. Rev. D49, 6636 (1994); ibid D52, 6770 (1995); B. L. Hu and A. Matacz, Phys. Rev. D51, 1577 (1995); A. Matacz, Phys. Rev. D55, 1860 (1997).

[10] D. Boyanovsky, R. Holman and S. Prem Kumar, Phys. Rev. D56, 1958 (1997).

[11] A. Albrecht and R. H. Brandenberger, Phys. Rev. D31, 1225 (1985); R. Brandenberger, H. Feldman and J. MacGibbon, Phys. Rev. D37, 2071 (1988); H. Feldman, Phys. Rev. D38, 459 (1988).

[12] O. I. Bogoyavlensky, "Methods in the Qualitative Theory of Dynamical Systems in Astrophysics and Gas Dynamics", Springer-Verlag (1985).

[13] V. A. Belinskii, L. P. Grishchuk, Ya. B. Zel'dovich and I. M. Khalatnikov, Zh. Eksp. Teor. Fiz. 89, 346 (1985) [Sov. Phys. JETP 62, 195 (1985)].

[14] V. A. Belinskii and I. M. Khalatnikov, Zh. Eksp. Teor. Fiz. 93, 784 (1987) [Sov. Phys. JETP 66, 441 (1987)].

[15] A. V. Nesteruk, R. Maartens and E. Gunzig, Portsmouth Univ. Preprint PU-RCR97-3, astro-ph/9703137. 
[16] H. P. de Oliveira, I. D. Soares and T. J. Stuchi, Phys. Rev. D56, 730 (1997); N. J. Cornish and J. J. Levin, Phys. Rev. D53, 3022 (1996); E. Calzetta and E. El Hasi, Phys. Rev. D51, 2713 (1995); G. Francisco and G. E. Matsas, Gen. Rel. Grav. 20, 1047 (1988). 


\section{Figure Captions}

Figure 1: The field trajectories in phase space (projected in the plane $x, z$ ) for the cases: (a) no dissipation; (b) constant dissipation ( $\beta_{0}=0.3$ for dashed line, $\beta_{0}=0.7$ for full line) and (c) field dependent dissipation $\left(\beta_{2}=10\right.$ for full line and $\beta_{2}=90$ for dashed line). Initial conditions for both cases are $\left(x_{0}, y_{0}, z_{0}\right)=\left(\sqrt{6}, 10^{-3}, 2.5\right)$. Dotted lines represent the lines where $\rho_{\text {rad }}=0$.

Figure 2: View of the physical region of the phase space. The infinity is compactified onto the plane $w=0$.

Figure 3: Phase portrait of the plane $w=0$ for the case of constant dissipation $(n=0)$. The inflationary domain is represented as the projection into the plane $w=0$.

Figure 4: Plot of $\frac{\ddot{a}}{a}$ against time, in the units of (2.8), for the cases of: no dissipation (full line); constant dissipation $\left(\beta_{0}=10\right)$, dashed line; and field dependent dissipation $\left(\beta_{2}=10 / 6\right)$, dotted line. Initial conditions for both cases are $\left(x_{0}, y_{0}, z_{0}\right)=\left(\sqrt{6}, 10^{-3}, 2.5\right)$.

Figure 5: Integral curves at infinity for the case of field dependent dissipation. The dashed circle represents the projection of the cone $\rho_{\text {rad }}=0$. The projection of the inflationary domain, into the plane $w=0$, is the same as in Fig. 3.

Figure 6: $\rho_{\text {rad }}$ for different values of dissipation. The mark indicates the exit of the inflationary stage. Initial conditions are $\left(\phi_{i}, \dot{\phi}_{i}, H_{i}\right)=\left(3.4 M_{\mathrm{pl}}, 0,3.4 \sqrt{\frac{4 \pi}{3}} m\right)\left(\rho_{\text {rad }_{i}}=0\right)$. 
FIGURE 1a

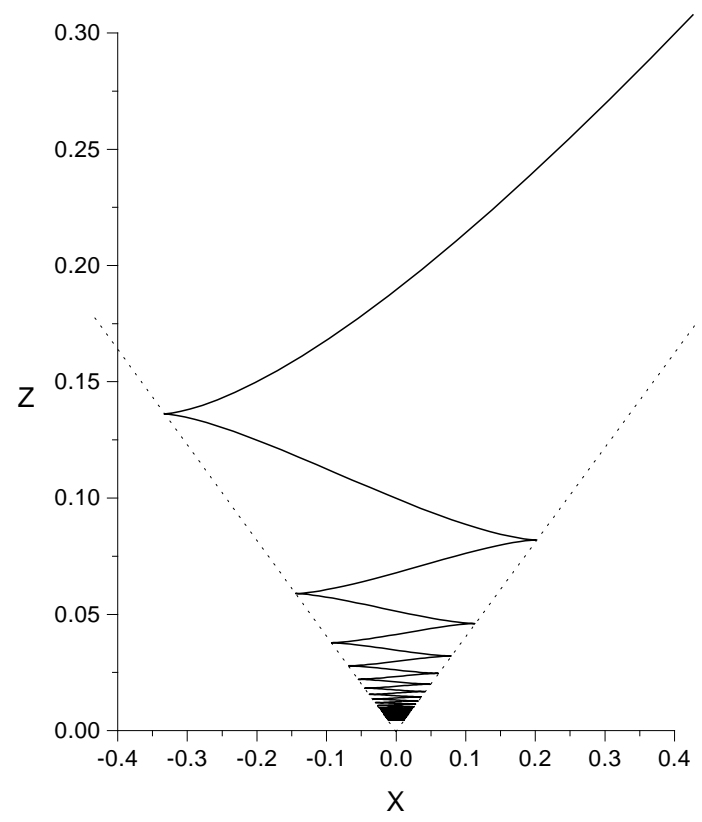

FIGURE $1 \mathrm{~b}$

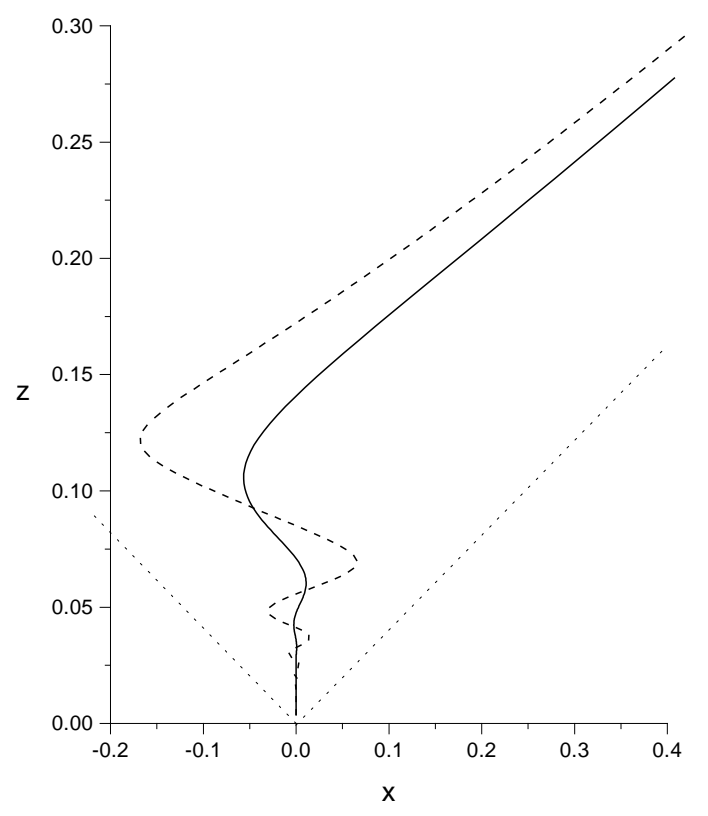

FIGURE 1C

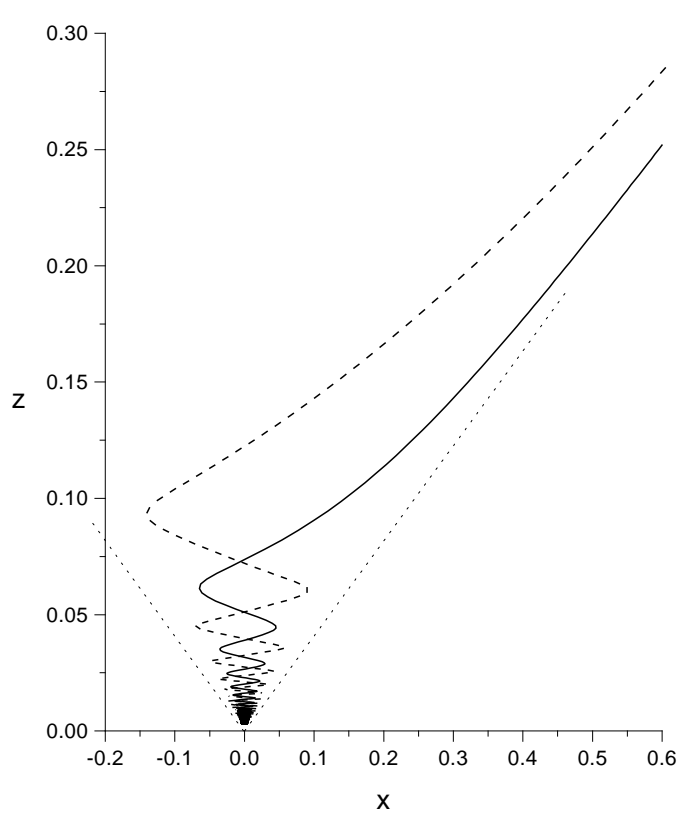


FIGURE 2

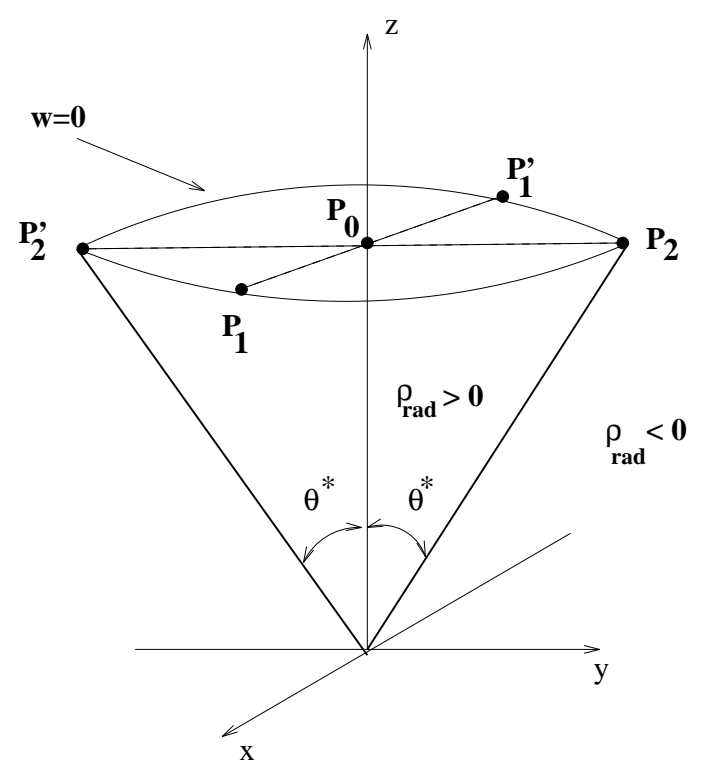

FIGURE 3

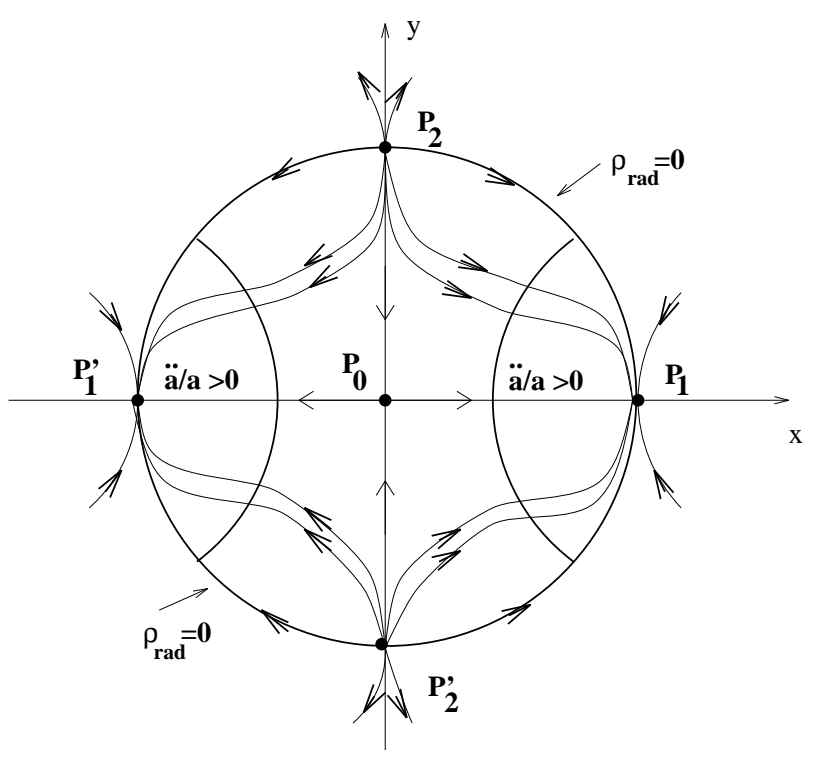


FIGURE 4

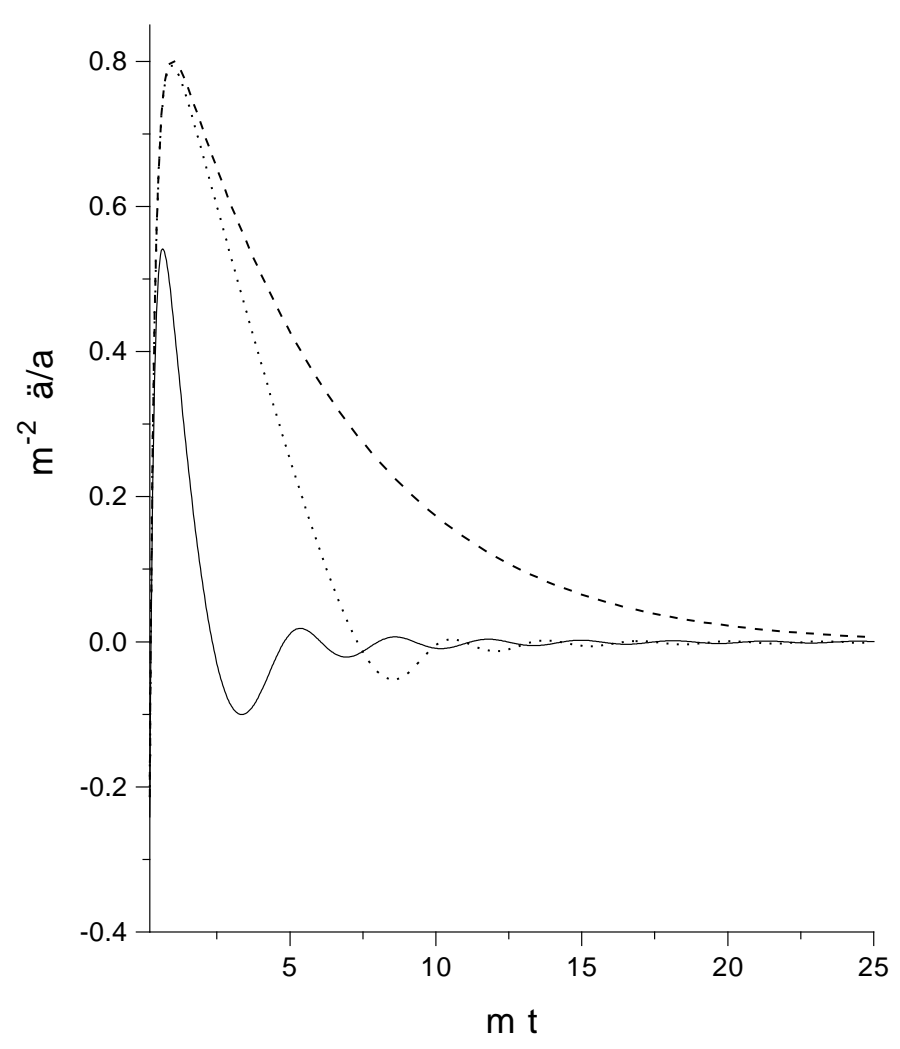

FIGURE 5

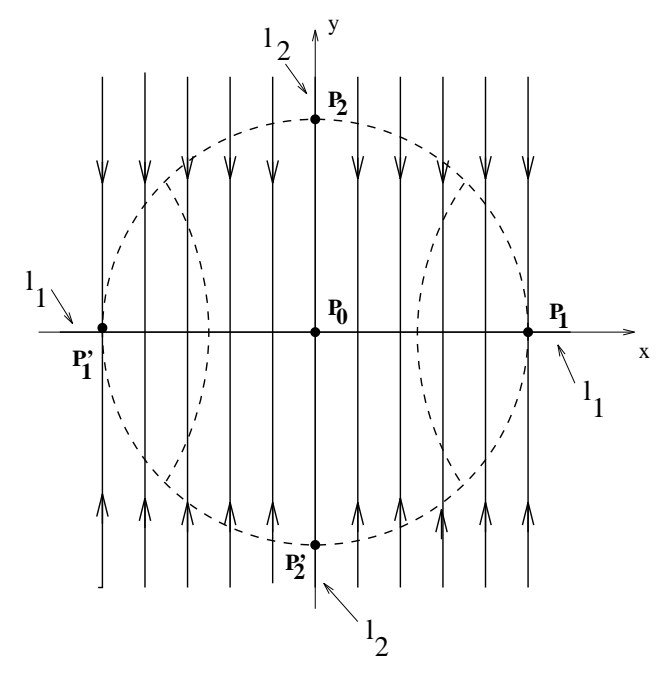


FIGURE 6

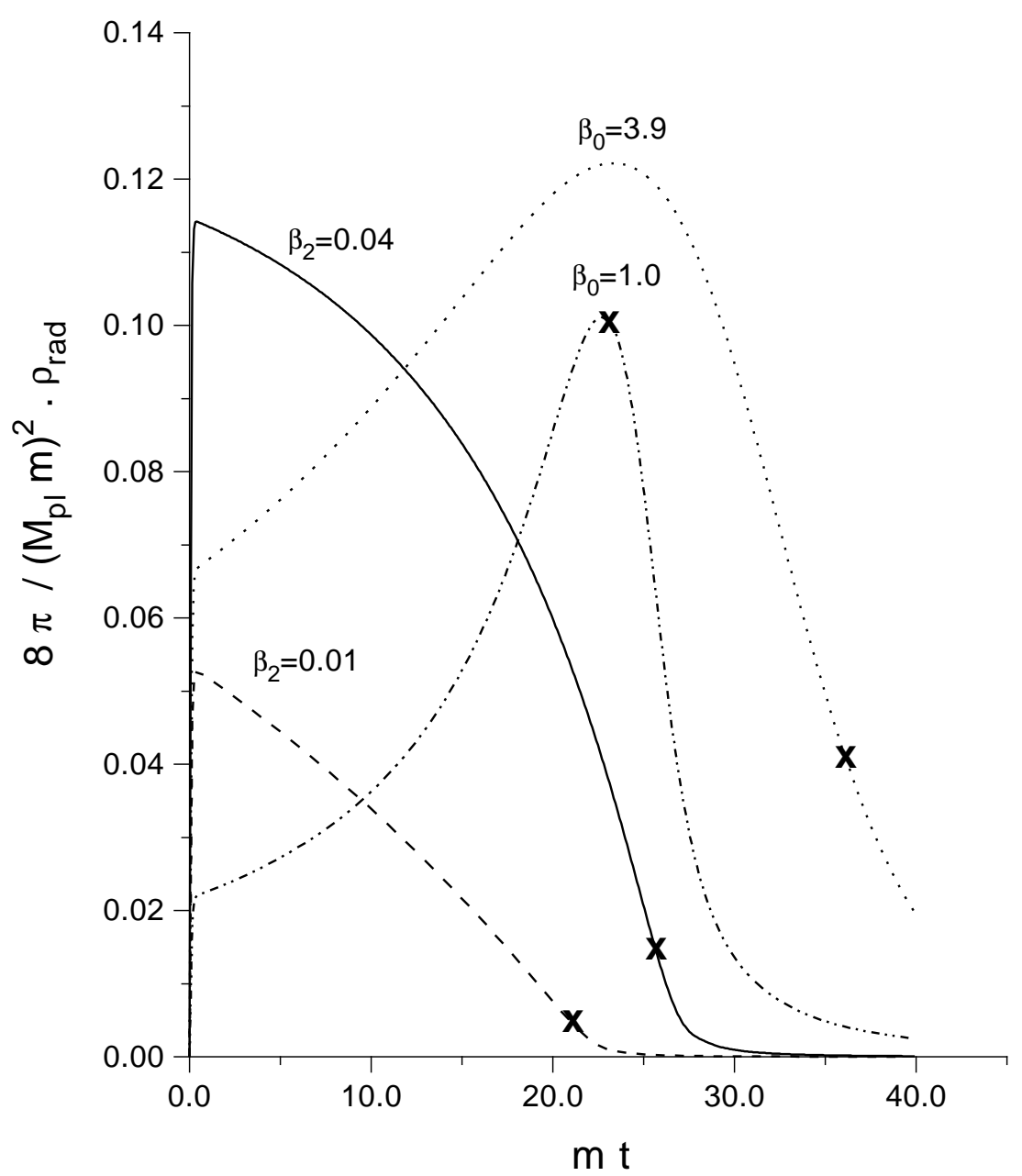

